\title{
Experimental evolution in Chlamydomonas. IV. Selection in environments that vary through time at different scales
}

\author{
REES KASSEN* \& GRAHAM BELL \\ Department of Biology and Redpath Museum, McGill University, 1205 avenue Dr Penfield, Montréal, Québec, \\ Canada H3A 1B1
}

\begin{abstract}
Specialists and two kinds of generalist were selected for in a genetically heterogeneous base population of the unicellular chlorophyte Chlamydomonas reinhardtii. The selection environments consisted of alternating periods of light and dark. When the environment remains constant (light or dark), specialists are expected to evolve; when the environment varies through time, generalists are expected to evolve. The kind of generalist that evolves depends on the period of environmental variation: versatile generalists capable of reversible responses to growth conditions are expected to evolve when the environment is fine-grained, whereas plastic generalists that respond irreversibly to the conditions of growth are expected to evolve when the environment is coarse-grained. The results indicate that specialists evolve in constant environments and generalists evolve in variable environments, as expected, but no evidence was found to support the idea that versatility and plasticity evolve in fine-grained and coarsegrained environments respectively. Moreover, the evolved generalists performed well in every environment and were insensitive to environmental variation. These results are interpreted to mean: (1) selection in the variable environments acted on the mean performance in each environment, rather than on the variance in performance across environments; (2) there was little cost to being a generalist.
\end{abstract}

Keywords: Chlamydomonas reinhardtii, environmental grain, generalist, plasticity, specialist, versatility.

\section{Introduction}

The terms 'specialist' and 'generalist' describe two ends of a spectrum of adaptation: at one extreme are specialists, which tolerate a narrow range of environmental conditions, whereas at the other are generalists, which tolerate a much broader range. In this paper, we present the results of an experiment designed to investigate how such differences in the breadth of adaptation evolve. More specifically, our experiment addresses the following questions: (1) under what environmental conditions do specialists and generalists evolve?; (2) How does the temporal scale of environmental variation affect the evolution of generalists?; (3) What prevents the evolution of a universally superior generalist?

*Correspondence. E-mail: rkassen@bio1.lan.mcgill.ca
When do specialists and generalists evolve?

The simplest theory of the evolution of specialists and generalists is that specialists should evolve when the environment remains constant through time, whereas generalists should evolve when the environment varies through time (see the general review by Futuyma \& Moreno, 1988). Specialists evolve in a temporally constant environment because selection favours types whose fitness is highest in that environment. Generalists, on the other hand, evolve in environments that vary through time because every type is compelled to grow in several environments, each of which may differ in the direction in which selection is operating; the type that evolves is one that has the highest fitness over all environments.

Note that this theory does not require that generalists be 'jacks-of-all-trades and masters of none'; there need not be a trade-off between ecological 
breadth and mean fitness (although this possibility has been raised by some authors; reviewed in Futuyma \& Moreno, 1988; Sultan, 1992; see also Van Tienderen, 1991). Nor does the theory specify the kinds of adaptations that will be favoured in either situation, although these have been dealt with in some theoretical treatments (for example Lloyd, 1984; Moran, 1992; Padilla \& Adolph, 1996). The central issue is how fitness is maximized when the environment is more or less variable through time (see, for example, Lynch \& Gabriel, 1987).

\section{Scales of temporal variation and the evolution of versatility and plasticity}

Environmental change through time can occur on at least two different scales relative to the lifetime of an individual. As an illustration, consider Fig. 1. On the left-hand side are three generations of a lineage of vegetatively reproducing unicells. On the right-

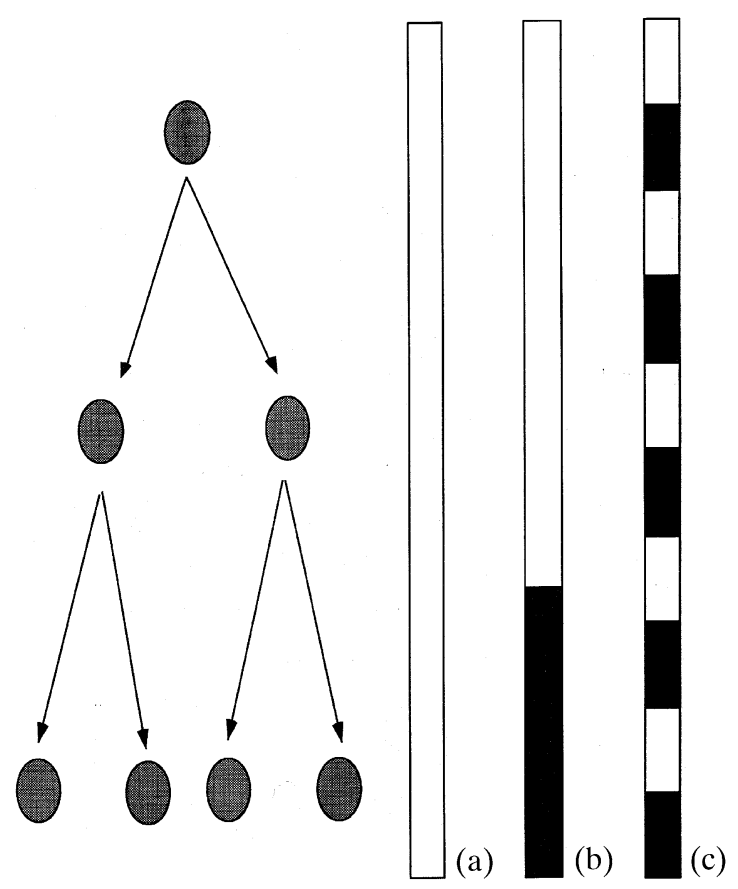

Fig. 1 Environmental variation at different time-scales relative to the lifetime of an individual. The solid white bar (a) represents an environment with one patch that remains constant through time; (b) represents a coarsegrained environment in which the environment consists of a series of patches that alternate once every few generations; (c) represents an environment that is fine-grained, the patches alternating within the lifetime of an individual. (a) favours the evolution of specialists; (b) and (c) favour the evolution of versatile and plastic generalists, respectively. hand side are three environments that alternate between two states ('white' and 'black') at three different scales: the environment may never change (Fig. 1a), it may change every few generations (Fig. $1 b)$ or it may change repeatedly within the lifetime of an individual (Fig. 1c). In Fig. 1a, only the white state is experienced in every generation, so selection acts consistently in the same direction and the lineage should evolve to become specialized to that state; Fig. 1(b,c) experience both white and black states with equal frequency, so selection repeatedly changes direction and this should lead to the evolution of generalism. What distinguishes Fig. 1b from Fig. $1 \mathrm{c}$ is the time-scale on which change occurs; Fig. $1 \mathrm{~b}$ is called coarse-grained variation and Fig. 1c finegrained variation, terms first coined by Levins (1968). Under coarse-grained variation (Fig. 1b), each individual experiences a single environmental state, although individuals living at different times may experience different states - the environment is 'patchy' in time. Fig. 1c depicts fine-grained variation, a situation in which every individual experiences both states, and individuals in subsequent generations experience the same series of states.

The dynamics of selection in fine-grained and coarse-grained environments depends on how the short-term fitness of an individual within a generation is related to the long-term fitness of the lineage as a whole (Levins, 1968; Lloyd, 1984; Gomulkiewicz \& Kirkpatrick, 1992). Assuming that individuals acquire resources additively within a patch, then when the environment is fine-grained, the overall fitness of an individual is the arithmetic average of its fitness in all the patches it experiences. Because each generation experiences the same set of patches, the fitness of a lineage in the long-term is the same as its average fitness among patches in the shortterm. This is not true in coarse-grained environments in which each generation does not necessarily experience the same type of patch. Here, any variance in fitness among patches reduces the long-term geometric mean fitness below that of the short-term arithmetic average fitness (for detailed discussions of the relationship between arithmetic and geometric mean fitness, see Gillespie, 1973; Bulmer, 1994; Bell, 1997).

These different scales of variation can also influence the kinds of adaptations that are favoured. In fine-grained environments, each generation experiences the same set of patches as every other generation, so the fittest type is one that is versatile; its phenotype should change appropriately as conditions change during its lifetime. Examples of versatile characters (also called labile characters by 
Scheiner, 1993) are inducible enzyme systems (e.g. Nguyen et al., 1989) and animal behaviour (Komers, 1996). In a coarse-grained environment, the fittest type is plastic; its phenotype is influenced by the state of the environment but, once determined, does not change afterwards. Examples of plastic characters are gender in species with environmental sex determination (Charnov, 1982) or alternative morphologies in species with heteroeocious life cycles (Moran, 1988).

The outcome of selection in variable environments is therefore expected to be the evolution of different kinds of generalist, as a consequence of the scale at which the environment changes through time. This amounts to saying that the variance of the environment through time is itself an environmental factor independently of the states of the patches considered separately; lineages may adapt to particular patterns of environmental change rather than, or in addition to, adaptation to particular environmental states. There is no experimental support for this conclusion as yet, although at least two experiments have shown that generalism evolves when temporal change is coarse-grained (bacteria, Bennett et al., 1992; Chlamydomonas, Reboud \& Bell, 1997).

\section{Costs of adaptation prevent the evolution of superior generalists}

If versatility and plasticity are specific adaptations to different scales of environmental variation, they can be interpreted in the same way as any other adaptation. This means that it should be possible to detect a cost of adaptation not only in specialists adapting to particular patches in an environment, but also in generalists adapting to different patterns of change.

There are two sources of a cost of adaptation: antagonistic pleiotropy and mutation accumulation. Both lead to negative genetic correlations in fitness across environments, but for different reasons. Antagonistic pleiotropy occurs when genes that improve fitness in one environment are detrimental in another. Mutation accumulation, on the other hand, occurs because mutations that are neutral in one environment are likely to be detrimental in another. Over time, selection will erode the antagonistic effects of pleiotropic gene action, although this will be balanced by the continuing accumulation of conditionally deleterious mutations. Which component makes the greater contribution to the overall cost of adaptation will depend on how genetic effects are correlated and on how long selection acts. Reboud \& Bell (1997) have discussed which components are responsible for the cost of adaptation in specialists and generalists. For now, it is sufficient to note that the existence of a cost of adaptation guarantees that a universally superior specialist or generalist should never evolve; adaptation will always be environment-specific, regardless of whether the environment is constant or varying through time.

\section{Materials and methods}

The general biology of Chlamydomonas reinhardtii and descriptions of the laboratory methods used in this experiment can be found in The Chlamydomonas Sourcebook (Harris, 1989) and in previous papers describing experimental evolution in C. reinhardtii (for example Reboud \& Bell, 1997, and references therein).

\section{Constructing the base population}

The base population was a genetically heterogeneous mixture consisting of the parents, $F_{1}$, backcross $\left(F_{1} \times\right.$ parents $)$ and $F_{2}$ progeny of matings between three mating type + strains and three mating type - strains $(\mathrm{CC} 1010+, \mathrm{CC} 1871+$, F94-16 + , CC410 - , CC1009- and CC1952-). All are standard laboratory strains that have been in culture for many years except for F94-16, which was isolated from a cornfield in Farnham, Québec in 1994.

\section{Selection environments}

The two different environmental states or patches in our experiment were light and dark. Chlamydomonas reinhardtii normally grows vegetatively as a photoautotroph in the light but can also grow as a heterotroph in the dark when given acetate as a carbon source (Harris, 1989). A strong negative genetic correlation between these two modes of growth has been shown to evolve in clonal populations after approximately 275 generations of selection in one or the other environment (Bell \& Reboud, 1997), which suggests that autotrophy and heterotrophy require different physiological competences. We selected for specialists by maintaining populations in either a constant light environment (light specialists) or a constant dark environment (dark specialists). Versatile generalists were selected in a fine-grained environment, which was constructed by alternating light and dark every hour (about five alternations within every generation). 
Plastic generalists were selected in a coarse-grained environment in which the light and dark conditions alternated every $24 \mathrm{~h}$ (about once every five generations). Each environment was located in a separate growth chamber in the McGill University Phytotron, except for the two specialist lines, which shared the same growth chamber. Dark conditions were created by maintaining the cultures in an opaque box.

\section{Maintaining the selection lines}

The lines were maintained on agar plates containing Bold's medium with $1.2 \mathrm{~g} \mathrm{~L}^{-1}$ sodium acetate as an additional carbon source. Every 3 or 4 days (every 7 days in the case of the dark lines, which grow much more slowly than the others) approximately $1.5 \times 10^{5}$ cells were transferred by washing the cells off the surface of the agar and transferring an aliquot $(250 \mu \mathrm{L})$ by pipette to a fresh plate. The experiment lasted for 10 weeks, representing roughly 400 generations in constant light, 150-200 generations in the fine-grained and coarse-grained environments and 80 generations in constant dark.

Three replicates of the base population were kept in the laboratory under dim illumination on plates containing identical medium and wrapped in paraffin wax. The stored base population was transferred once and underwent no more than eight generations during the course of the experiment.

Three replicate lines within each treatment (selection environment) were arranged as a randomized block design within each environment chamber. Block effects were significant $\left(F_{2,45}=9.10\right.$, $P=0.0005)$, so all the analyses and data presented in the paper have been adjusted for these effects.

\section{Measuring the effects of selection}

Assay procedure We grew samples from each selection line and the base population in every environment. The assay plates received an inoculum the same size as those used in routine transfers. The assay thus comprised (four selection lines + base population $) \times($ four environments $) \times($ three replicates $)=60$ plates. These were laid out in the growth chambers in the same manner as the selection lines.

Fitness We used the initial growth rate of colonies as our measure of fitness, which we estimated by calculating the mean number of divisions of 100 colonies on a plate $24 \mathrm{~h}$ after it was inoculated. This provides an estimate of $r_{\max }$, the intrinsic rate of growth in pure culture, which should be a good esti- mate of fitness in competition as long as nutrients remain unlimiting (see Bell, 1997, for a more detailed discussion). Our selection lines were transferred often enough to ensure that most lines were maintained near their maximum rate of increase throughout the experiment.

This procedure differed slightly for assays conducted in the coarse-grained environment, in which one cycle of growth (five generations in the light, five generations in the dark) comprised $24 \mathrm{~h}$ in the light and then $24 \mathrm{~h}$ in the dark. In order to ensure that separate colonies could be distinguished, lines assayed in the coarse-grained environment necessarily experienced less than one full growth cycle. As a result, not all lines assayed in the coarsegrained environment experienced the same photoperiod, although all experienced at least one full generation in either the light or the dark. To account for these differences in photoperiod, we adjusted by regression the growth rates of lines assayed in the coarse-grained environment to a $12 \mathrm{~h}$ light $/ 12 \mathrm{~h}$ dark photoperiod.

Adaptation The difference in fitness between a selection line and the base population was calculated as a measure of adaptation. A positive value indicates that a selection line grew faster than the base population in a particular environment; a negative value indicates that it grew more slowly.

\section{Results}

The general response to selection

The results of selection are given in Table 1 and shown graphically in Fig. 2 . The values represent the difference in growth rate from the base population. All selection lines grew faster than the base population. There were significant differences in the response to selection among selection lines, among assay environments and an interaction of the two, indicating that the lines diverged substantially because of selection in different environments (Table 2). The synclinal response to selection in all lines suggests that part of the response observed was caused by a general adaptation to the conditions of the growth chambers.

\section{Selection for specialists}

The line selected in the light grew better when tested in the light than in the dark; the reverse was true for the dark-selected line (Fig. 2). This supports the idea that selection in constant environments 
Table 1 Difference in growth rate of each selection line from the base population when assayed in every environment

\begin{tabular}{lccccc}
\hline & & \multicolumn{4}{c}{ Assay environment } \\
\cline { 3 - 6 } Selection line & Replicate & Light & Dark & Fine & Coarse \\
\hline Light specialist & 1 & $\mathbf{1 . 1 9 5}$ & 0.408 & 0.771 & 1.370 \\
& 2 & $\mathbf{1 . 0 3 8}$ & 0.463 & 0.831 & 1.757 \\
Dark specialist & 3 & $\mathbf{1 . 3 7 8}$ & 0.088 & 0.648 & 1.124 \\
& 1 & 0.205 & $\mathbf{0 . 8 7 8}$ & 0.381 & 0.370 \\
Fine-grained generalist & 2 & 0.988 & $\mathbf{1 . 0 0 3}$ & 0.561 & 0.267 \\
& 3 & 0.728 & $\mathbf{0 . 9 3 8}$ & 0.498 & 0.364 \\
Coarse-grained generalist & 1 & 1.385 & 0.628 & $\mathbf{0 . 7 5 1}$ & 1.640 \\
& 2 & 1.278 & 0.733 & $\mathbf{0 . 6 8 1}$ & 1.567 \\
& 3 & 1.008 & 0.538 & $\mathbf{1 . 0 7 8}$ & 1.834 \\
& 1 & 1.435 & 0.478 & 0.901 & $\mathbf{0 . 6 8 0}$ \\
& 2 & 0.918 & 0.193 & 0.731 & $\mathbf{0 . 4 6 7}$ \\
& 3 & 1.108 & 0.828 & 0.578 & $\mathbf{0 . 7 3 4}$ \\
\hline
\end{tabular}

Values have been adjusted for the variable photoperiod in the coarse-grained environment and for block effects. Numbers in bold indicate lines selected and assayed in the same environment.

leads to specialization associated with a cost of adaptation.

\section{Selection for generalists}

Selection for generalization was less successful than selection for specialization (Fig. 2). There was no clear indication that the two generalist lines did better than all others when tested in their own environments, but they did little worse than the specialist lines when tested in the constant environments. The lines selected in coarse-grained and finegrained environments seem, therefore, to have adapted to both light and dark conditions of growth,

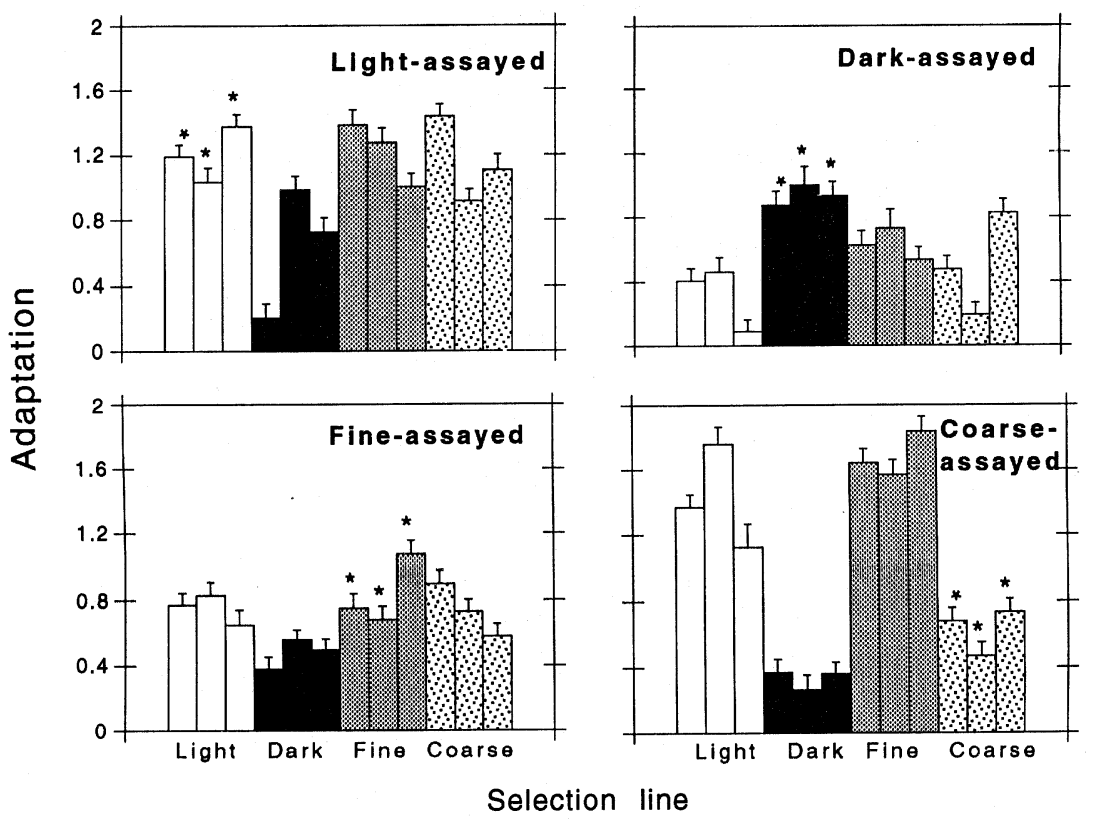

Fig. 2 Responses to selection of lines assayed in the four environments. All three replicates are shown. Error bars represent the standard error among colonies within a plate. Lines selected and assayed in the same environment are denoted by an asterisk $\left(^{*}\right)$.

(C) The Genetical Society of Great Britain, Heredity, 80, 732-741. 
Table 2 Two-way ANOva treating selection line and assay environment as factors

\begin{tabular}{lrrrrr}
\hline Source & d.f. & SS & MS & $F$ & $P$ \\
\hline Selection line & 3 & 1.802 & 0.601 & 10.540 & 0.0001 \\
Assay environment & 3 & 2.200 & 0.740 & 12.990 & 0.0001 \\
Selection line $\times$ assay environment & 9 & 3.764 & 0.418 & 7.34 & 0.0001 \\
Residual & 30 & 1.709 & 0.057 & & \\
\hline
\end{tabular}

Values have been adjusted for variable coarse-grained photoperiod and block effects.

without evolving plasticity or versatility as a response to specific patterns of environmental change.

\section{The response to selection expressed as progress in} the light and dark

Figure 3 shows the amount of adaptation that has occurred in the selection lines according to their performance in the two constant environments, light and dark. Selection for specialization leads to a negative genetic correlation for growth in the two environments. The two generalist lines fall above the line connecting the light specialists and the dark specialists, indicating that selection has improved performance in each component patch (light and dark) of the variable environments. Evidently, there is little cost to being a generalist in the short-term, regardless of the scale of environmental variability.

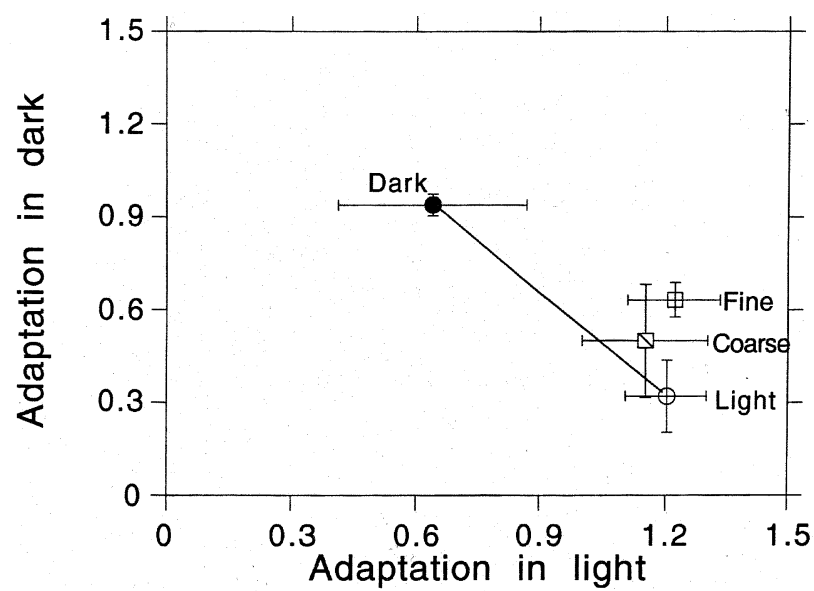

Fig. 3 Adaptation to the light and dark components of each environment. The light specialist does better in the light and than in the dark. The reverse is true for the dark specialist. The two generalist lines do equally as well as the light specialist in the light and slightly better than the light specialist in the dark. Error bars are the standard error of the three replicates.
The response to selection expressed as sensitivity to different environments

The sensitivity of a selection line is a measure of its ability to maintain its fitness across a range of environments (Falconer, 1990; reaction norm sensu Stearns, 1989) and can be measured by calculating the slope of the line relating performance (rate of division) against a range of environments (photoperiod). A steep slope indicates that the character is sensitive to changes in the environment and a shallow slope that it is insensitive. (Note that, when there are more than two environments, as in our experiment, a polynomial function may describe the sensitivity of a line better. Fitting a polynomial to our data did not improve the fit substantially, so we report only the slope of the linear regression, which is conceptually and biologically more straightforward; see Via et al., 1995.)

In our experiment, selection increased the sensitivity of all lines except the dark-selected line (Fig. 4). An analysis of covariance showed that the interaction between photoperiod and response to selection was highly significant, indicating that lines diverged in their sensitivity according to the selection environment (Table 3). The light-selected line had the highest sensitivity and the dark-selected line the lowest. The two types of generalist lines had sensitivities intermediate between these two extremes.

Figure 5 depicts the sensitivity of the four selection lines as a function of their performance in the light and in the dark. If there were a large cost to being a generalist, then we would expect that low sensitivity would be associated with fitnesses that are intermediate between that of the specialists in each environment. This is not the case: the two generalist lines had lower sensitivities than the light-selected line but their performance was nearly equal to the light-selected line in the light and superior to it in the dark. The differences in sensitivity between the two generalist lines and the light-selected line were significant (ANCOVA; $F_{3,28}=8.96, P=0.0003$ ). 


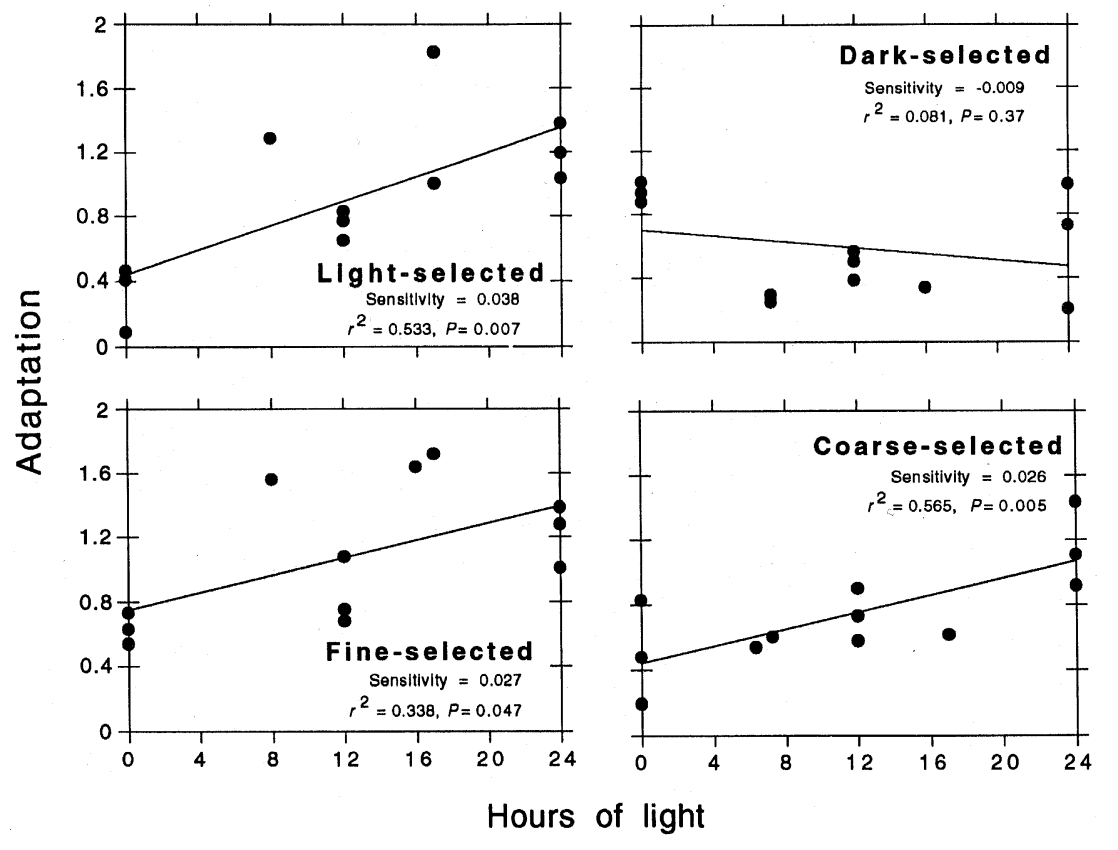

Fig. 4 Sensitivity of the selection lines to changes in photoperiod. The light line was the most sensitive, the dark line the least sensitive. The generalist lines were intermediate between the two. The base population is located at the origin. Sensitivity is the slope of adaptation against photoperiod. Probability values are for the ANCOVA between adaptation and photoperiod.

\section{Discussion}

The evolution of specialists and generalists

Selection in the two constant environments was generally antagonistic: adaptation to one environment was coupled to regress in the other. Selection in the variable environments was not, however. The fine-grained and coarse-grained lines grew as well in the light as the line selected in the light and almost as well in the dark as the line selected in the dark. These results confirm those of previous experiments (Bennett et al., 1992; Leroi et al., 1994; Bell \& Reboud, 1997) and lend further experimental support to the idea that selection in environments that remain constant through time leads to the evolution of specialization, and selection in environments that vary through time leads to the evolution of generalization. Moreover, it provides some insight into a previously untested aspect of the theory of selection in variable environments: selection at a fine-grain scale produces ecologically the same result as selection at a coarse-grain scale, namely, generalism. If this result can be extended, it might imply that selection for versatility in agronomic trials might be an effective way of increasing the plasticity of crop plants.

\section{The evolution of versatility and plasticity}

The generally antagonistic nature of adaptation in constant environments is not surprising. Selection in one direction for prolonged periods of time is thought to involve a cost because of the accumulation of conditionally deleterious mutations, the fixation of genes with pleiotropic effects that are antagonistic in novel environments or some

Table 3 Analysis of covariance treating selection line and hours of light as covariates

\begin{tabular}{lrccrc}
\hline Source & d.f. & SS & MS & \multicolumn{1}{c}{$F$} & $P$ \\
\hline Hours of light & 1 & 1.555 & 1.555 & 15.82 & 0.0003 \\
Selection line & 3 & 0.344 & 0.115 & 1.17 & 0.3344 \\
Selection line $\times$ hours of light & 3 & 1.186 & 0.396 & 4.03 & 0.0136 \\
Residual & 40 & 3.932 & 0.098 & & \\
\hline
\end{tabular}

Growth rate values are adjusted for replicate effects but not coarse-grained photoperiod effects. 
combination of the two. Indeed, adaptation was generally antagonistic for the dark specialist. It grew less well than any other line when tested in any environment save its own. The same was not true for the light specialist, which grew as well as the two generalist lines in the generalist environments. This suggests that the cost to adaptation is not necessarily symmetrical, although it might become more symmetrical if selection were continued for longer.

The negative genetic correlation of light and dark growth shown by the specialist lines did not appear in the generalists. Both the fine-grained line and the coarse-grained line did almost as well in the light as did the light specialist itself. Moreover, they did better in the dark than the light specialist, indicating that at least some adaptation had occurred to growth in the dark as well, although not as much as

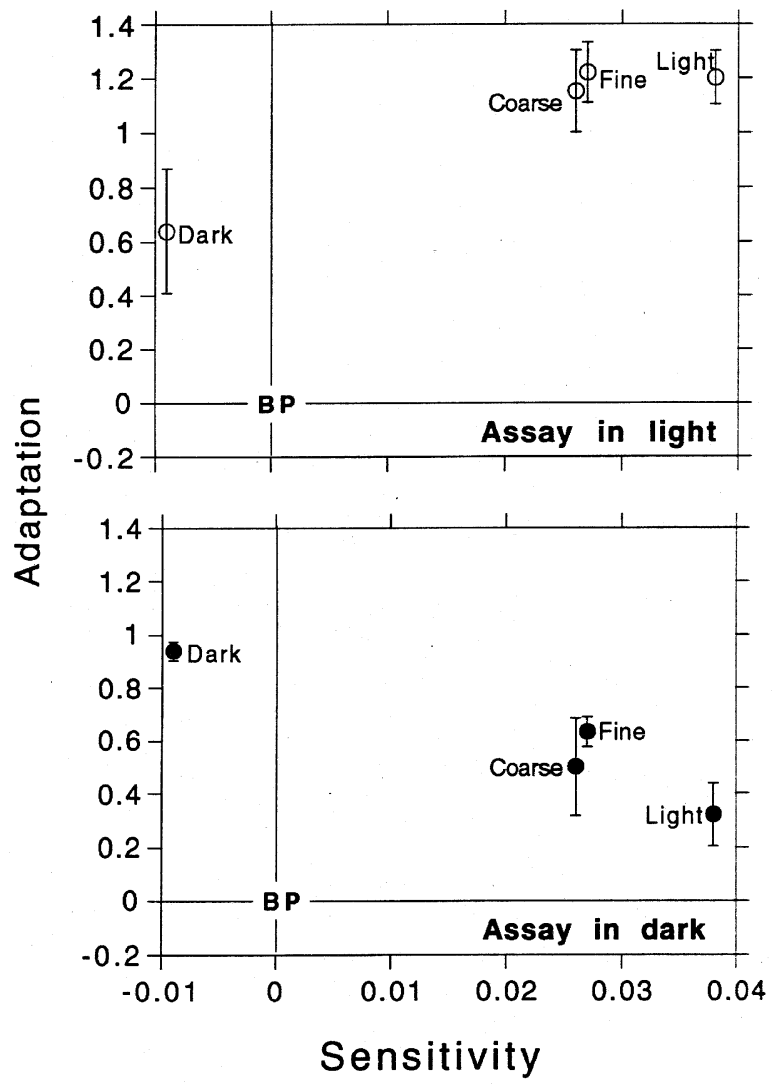

Fig. 5 The relationship between sensitivity and adaptation to the light (top) and the dark (bottom). The averages of the three replicates are shown. The base population (BP) is located at the origin. The two generalists were relatively less sensitive to environmental variation than the light specialist. The generalists grew at nearly the same rate as the light specialist in the light and faster than the light specialist in the dark. in the lines selected only in the dark. However, neither of these lines had the highest fitness in their own selection environment, suggesting that adaptation to the pattern of environmental change requires specific adaptations (versatility when the environment is fine-grained and plasticity when it is coarsegrained) that were not observed in this experiment. Generalization seems instead to have evolved mainly through adaptation to each patch of the environment, a result consistent with experiments by Leroi et al. (1994). This lends empirical support to the view that generalism evolves through selection on character means, rather than on the reaction norm itself (see Via et al., 1995).

\section{Costs of adaptation and the evolution of superior generalists}

It is conceivable that the adage 'jack-of-all-trades is a master of none' might describe the relationship between the performance of specialists and generalists in constant environments; specialists having high performance in their own environment and low performance elsewhere and generalists having mediocre performance in every environment. This in turn implies that specialists would be more sensitive to environmental variation than generalists. Evidently this is not the case. The less sensitive generalists perform almost as well as the light specialist in the light and better than them in the dark. In the short-term at least, selection for generalization can lead to types that are both insensitive to environmental variation and have high performance across a range of environments. Thus, selection may lead to the evolution of a superior generalist type whenever the environment varies through time. What is it, then, that restricts the evolution of generalism?

In the long-term, it seems likely that there must be a cost to adaptation, and our experiment may not have been continued for long enough to be able to detect it. Indeed, the existence of a variety of behavioural, morphological and physiological phenomena at many levels of biological integration is evidence that versatility and plasticity can evolve as unique traits. However, the fact that their evolution was not observed in our experiment argues either that no variation for the relevant characters exists in our population or that evolution of these traits requires concerted and persistent selective pressures over long periods of time. One way of testing this idea is to use artificial selection for versatility and plasticity by selecting directly or indirectly on the 
environmental variance. We intend to set up experiments of this kind.

An alternative idea, put forward by Whitlock (1996), is that the appropriate trade-off is between the rates at which specialists and generalists evolve. When the environment is constant, beneficial alleles go to fixation faster and deleterious ones are lost faster than when the environment is variable. Thus, specialists may evolve faster than generalists because their environment is persistent, whereas the environment of a generalist is not. Our results provide some circumstantial support for this idea. The dark specialist was superior to all other lines in the dark after just 80 generations of selection, despite the fact that selection had occurred for almost twice as long in the two generalist lines.

These two explanations need not be mutually exclusive. Indeed, a trade-off between the rates at which specialists and generalists evolve seems likely, at least in the short-term, considering that genetic correlations can prevent a population from achieving the optimal phenotype in any given environment (Via \& Lande, 1985; Gomulkiewicz \& Kirkpatrick, 1992). In the long-term, however, these correlations would be broken down by mutation, so a cost of adaptation must be ultimately responsible for preventing the evolution of universally superior generalists.

\section{Acknow ledgements}

We are grateful to M. Romer and C. Cooney for assistance with the growth chambers in the Phytotron, Y. Ducharme and S. Goho for technical assistance, D. Kramer and G. Stirling for discussion. This work was supported by a Natural Sciences and Engineering Research Council (NSERC) of Canada Research Grant to G.B. and an NSERC Postgraduate Fellowship to R.K.

\section{References}

BELL, G. 1997. Selection: the Mechanism of Evolution. Chapman \& Hall, Toronto.

BELL, G. AND REBOUD, x. 1997. Experimental evolution in Chlamydomonas. II. Genetic variation in strongly contrasted environments. Heredity, 78, 498-506.

BENNETT, A. F., LENSKI, R. E. AND MITTLER, J. E. 1992. Evolutionary adaptation to temperature. 1. Fitness responses of Escherichia coli to changes in its thermal environment. Evolution, 46, 16-30.

BULMER, M. 1994. Theoretical Evolutionary Ecology. Sinauer Associates, Sunderland, MA.
Charnov, E. L. 1982. The Theory of Sex Allocation. Princeton University Press, Princeton, NJ.

FALCONER, D. s. 1990. Selection in different environments: effects on environmental sensitivity (reaction norm) and on mean performance. Genet. Res., 56, 57-70.

FUtUYMA, D. J. AND MORENO, G. 1988. The evolution of ecological specialization. Ann. Rev. Ecol. Syst., 19, 207-233.

GILLESPIE, J. H. 1973. Natural selection with varying selection coefficients - a haploid model. Genet. Res., 21, $115-120$.

GOMULKIEWICZ, R. AND KIRKPATRICK, M. 1992. Quantitative genetics and the evolution of reaction norms. Evolution, 46, 390-411.

HARRIS, E. 1989. The Clamydomonas Sourcebook. Academic Press, New York.

KOMERS, P. 1996. Behavioural plasticity in variable environments. Can. J. Zool., 75, 161-169.

LEROI, A., LENSKI, R. E. AND BENNETT, A. F. 1994. Evolutionary adaptation to temperature. III. Adaptation of Escherichia coli to a temporally varying environment. Evolution, 48, 1222-1229.

LEVINS, R. 1968. Evolution in Changing Environments. Princeton University Press, Princeton, NJ.

LLOYD, D. G. 1984. Variation strategies of plants in heterogeneous environments. Biol. J. Linn. Soc., 21, 357-385.

LYNCH, M. AND GABRIEL, w. 1987. Environmental tolerance. Am. Nat., 129, 283-303.

MORAN, N. A. 1988. The evolution of host-plant alternation in aphids: evidence for specialization as a dead end. Am. Nat., 132, 681-706.

MORAN, N. A. 1992. The evolutionary maintenance of alternative phenotypes. Am. Nat., 139, 971-989.

NGUYEN, T. N. H., PHAN, Q. G., DUONG, L. P., BERTRAND, K. P. AND LENSKI, R. E. 1989. Effects of carriage and expression of the $\operatorname{Tn} 10$ tetracycline-resistance operon on the fitness of Escherichia coli K12. Mol. Biol. Evol., 6, 213-225.

PADIlla, D. K. AND ADOLPH, S. C. 1996. Plastic inducible morphologies are not always adaptive: the importance of time delays in a stochastic environment. Evol. Ecol., 10, 105- 117.

REBOUD, X. AND BELL, G. 1997. Experimental evolution in Chlamydomonas. III. Evolution of specialist and generalist types in environments that vary in space and time. Heredity, 78, 507-514.

SCHEINER, S. M. 1993. Genetics and evolution of phenotypic plasticity. Ann. Rev. Ecol. Syst., 24, 35-68.

STEARNS, s. C. 1989. The evolutionary significance of phenotypic plasticity. BioScience, 39, 436-445.

Sultan, S. E. 1992. Phenotypic plasticity and the neoDarwinian legacy. Evol. Trends Plants, 6, 61-71.

VAN TIENDEREN, P. H. 1991. Evolution of generalists and specialists in spatially heterogeneous environments. Evolution, 45, 1317-1331.

VIA, S. AND LANDE, R. 1985. Genotype-environment interaction and the evolution of phenotypic plasticity. Evolution, 39, 505-522.

(c) The Genetical Society of Great Britain, Heredity, 80, 732-741. 
VIA, S., GOMULKIEWICZ, R., DE JONG, G., SCHEINER, S. M., SCHLICHTING, S. M., AND VAN TIENDEREN, P. H. 1995. Adaptive phenotypic plasticity: consensus and controversy. Trends Ecol. Evol., 10, 212-217. whitlock, м. c. 1996. The Red Queen beats the jackof-all-trades: the limitations on the evolution of phenotypic plasticity and niche breadth. Am. Nat., 148, S65-S77. 